\title{
Bright attosecond $\gamma$-ray pulses from nonlinear Compton scattering with laser-illuminated compound targets
}

\author{
Xing-Long Zhu, ${ }^{1,2}$ Min Chen, ${ }^{1,2}$ Tong-Pu Yu, ${ }^{3}$ Su-Ming Weng, ${ }^{1,2}$ Li-Xiang Hu, ${ }^{3}$ Paul McKenna, ${ }^{4}$ and \\ Zheng-Ming Sheng ${ }^{1,2,4,5, a)}$ \\ ${ }^{1)}$ Key Laboratory for Laser Plasmas (MOE), School of Physics and Astronomy, Shanghai Jiao Tong University, \\ Shanghai 200240, China \\ ${ }^{2)}$ Collaborative Innovation Center of IFSA, Shanghai Jiao Tong University, Shanghai 200240, \\ China \\ 3) Department of Physics, National University of Defense Technology, Changsha 410073, \\ China \\ ${ }^{4)}$ SUPA, Department of Physics, University of Strathclyde, Glasgow G4 ONG, \\ $U K$ \\ 5) Tsung-Dao Lee Institute, Shanghai Jiao Tong University, Shanghai 200240, \\ China
}

Attosecond light sources have the potential to open up new avenues in ultrafast science. However, the photon energies achieveable using existing generation schemes are limited to the keV range. Here we propose and numerically demonstrate an all-optical mechanism for the generation of bright $\mathrm{MeV}$ attosecond $\gamma$-photon beams with desirable angular momentum. Using a circularly-polarized Laguerre-Gaussian laser pulse focused onto a cone-foil target, dense attosecond bunches $(\lesssim 170$ as $)$ of electrons are produced. The electrons interact with the laser pulse which is reflected by a plasma mirror, producing ultra-brilliant $\left(\sim 10^{23}\right.$ photons $\left./ \mathrm{s} / \mathrm{mm}^{2} / \mathrm{mrad}^{2} / 0.1 \% \mathrm{BW}\right)$ multi-MeV $\left(E_{\gamma, \max }>30 \mathrm{MeV}\right)$ isolated attosecond $(\lesssim 260$ as $) \gamma$-ray pulse trains. Moreover, the angular momentum is transferred to $\gamma$-photon beams via nonlinear Compton scattering of ultra-intense tightly-focused laser pulse by energetic electrons. Such brilliant attosecond $\gamma$-photon source would provide new possibilities in the attosecond nuclear science.

Ultra-short pulse radiation sources are powerful tools enabling microcosmic structures and ultrafast dynamical processes to be investigated with ultrahigh timespace resolution $^{1,2}$. Furthermore, recently the generation of attosecond pulses has given rise to attosecondscience research ${ }^{3,4}$, such as ultrafast probing and controlling sub-atomic-scale electronic and even nuclear dynamics. The interaction of ultrashort laser pulses with high energy electron beams provides a promising approach to produce ultrashort X-ray and even $\gamma$-ray radiation sources. Although high-order harmonic generation ${ }^{5-7}$ in laser-plasma interactions has been widely studied as a way to produce intense attosecond photon sources, the energy of the radiation is usually limited to the sub-keV level. Tunable, multi-MeV attosecond $\gamma$-ray sources are particularly desirable for a number of studies ${ }^{8,9}$, such as exploring nuclear structure, intranuclear dynamics and nuclear resonance fluorescence. In recent years, high energy radiation sources based-on Thomson (or Compton) scattering have been demonstrated experimentally, using laser-wakefield accelerated beams of electrons ${ }^{10-13}$. The photon energy achieved can be as high as several $\mathrm{MeV}$, delivered in a pulse with a duration of the order of tens-of-femtoseconds. It is difficult to produce attosecond photon pulses by this way because the radiated photon pulse duration is determined by the shortest duration of either the laser pulse or energetic electron bunch and both of them are generally beyond femtoseconds in duration.

a) Electronic mail: zmsheng@sjtu.edu.cn
In addition to the photon energy and pulse duration, the angular momentum of the photon beam is also an important property. Laser-driven energetic particle and photon beams with angular momentum possess an additional degree of freedom. This is of importance for many applications ${ }^{14-16}$, including investigation of the transfer of angular momentum between the photon and particle beams, exploration of the structure of particles, studies involving energetic rotating systems and fundamental physics research ${ }^{17,18}$. Although several mechanisms ${ }^{19-29}$ have been proposed to produce short, energetic $\gamma$-ray sources, the emitted $\gamma$-photon beams are unfavorable. They are either not of attosecond duration ${ }^{19-21}$, do no have angular momentum ${ }^{22,23}$, or both ${ }^{24-29}$. In addition, these approaches require either a multi-petawatt laser to achieve the extremely high intensity and/or an additional beam of high energy electrons. Bright attosecond $\gamma$-ray beams with tunable angular momentum, based-on alloptical compact laser-plasma interactions, have not yet been realized at presently available laser systems.

In this Letter, we present an all-optical laser-plasma scheme to efficiently produce multi-MeV $\gamma$-photon pulses with attosecond duration, ultrahigh brilliance and high tunable angular momentum. This is achieved in the quantum radiation-dominated regime by use of circularly-polarized (CP) laser pulse with a LaguerreGaussian (LG) mode and a target geometry which significantly enhances laser-electron scattering, as schematically illustrated in Fig. 1(a). Shaping the laser focus in this way opens up new degrees of control on particle acceleration and photon generation. When the drive laser pulse passes through the cone, it is focused with its inten- 
sity greatly enhanced, as shown in Figs. 1(b) and 1(c). In this sheme, a large number of electrons are periodically dragged out from the cone walls to form a train of dense attosecond electron bunches. In each of the bunch trains, electrons form a donut-shaped sheet with an ultra-short duration of hundreds of attoseconds [see Fig. 1(d)]. This is produced by the CP LG laser fields having unique components of electric fields in the radial and azimuthal directions, which are spatially periodic donut-shaped. This unique arrangemnet induces electron dynamics that is quite different from the case of a normal CP Gaussian laser-target interaction. In our scheme, the electrons can be efficiently accelerated in the longitudinal direction and gradually form isolated annular bunch trains with a separation of about one laser wavelength. The electron bunches then collide head-on with the ultraintense laser pulse reflected from the foil and nonlinear Compton Scattering (NCS) ${ }^{30,31}$ is triggered, resulting in dense isolated attosecond $\gamma$-photon pulse trains in the forward direction, as shown in Fig. 1(e). Furthermore, the angular momentum is transferred to the $\gamma$-photon beams via the NCS of such focused intense laser pulse by ultra-relativistic electron bunches.

In order to investigate the underlying physical processes, we have first performed full three-dimensional particle-in-cell (3D-PIC) simulations with the QED-PIC code $\mathrm{EPOCH}^{32}$, where the quantum-corrected photon emission has been incorporated ${ }^{33}$. In the simulation$\mathrm{s}$, the incident laser pulse has a spatial distribution of $a\left(\mathrm{LG}_{p}^{l}\right)=a_{0} C_{p}^{l}(-1)^{p} \exp \left(-r^{2} / \sigma_{0}^{2}\right)\left(\sqrt{2} r / \sigma_{0}\right)^{l} L_{p}^{l}\left(2 r^{2} / \sigma_{0}^{2}\right)$ $\exp (i l \phi)\left(\cos \psi \vec{e}_{y}+\sin \psi \vec{e}_{z}\right)$ and a trapezoidal temporal profile of $9 T_{0}\left(1 T_{0}-7 T_{0}-1 T_{0}\right)$, where $\sigma_{0}=3.5 \lambda_{0}$ is the laser focus spot size, $\lambda_{0}=c T_{0}=0.8 \mu \mathrm{m}$ is the laser wavelength, $\phi \in[0,2 \pi]$ is the azimuthal angle, $\psi$ is the laser phase term, $C_{p}^{l}$ is the normalized parameters, $l=1$ and $p=0$ implying the $\mathrm{LG}_{0}^{1}$ laser beam with a low-order (1, 0) mode. Such a laser pulse can be obtained using optical devices $^{34,35}$ or plasma-based optical media ${ }^{36,37}$. The normalized laser amplitude is $a_{0}=e E_{0} / m_{e} c \omega_{0}=15$ with a readily achievable pulse energy of $5 \mathrm{~J}$, where $e, m_{e}, c$ are the unit charge, the electron mass and the speed of light in vacuum, respectively. $\omega_{0}$ and $E_{0}$ are the laser frequency and the electric field amplitude, respectively. The size of the simulation box is $24 \lambda_{0}(x) \times 14 \lambda_{0}(y) \times 14 \lambda_{0}(z)$, sampled by $1440 \times 280 \times 280$ cells with 16 macro-particles per cell for the cone target and 48 macro-particles per cell for the foil. Absorption boundary conditions are employed for both particles and fields, and a moving window is used to save computing resources. The cone target has a density of $n_{0}=25 n_{c}$ (where $n_{c}=m_{e} \omega_{0}^{2} / 4 \pi e^{2}$ is the critical density) with a longitudinal axial length of $20 \lambda_{0}$ located between 3 and $23 \lambda_{0}$. The left and right aperture radius of the cone are $R_{0}=5 \lambda_{0}$ and $r_{0}=1.8 \lambda_{0}$, respectively. In addition, a thin solid aluminum foil with a density of $390 n_{c}$ is adopted as a plasma mirror with a thickness of $1 \lambda_{0}$.

Here the cone target acts as a plasma optical lens to focus the laser pulse ${ }^{38-40}$. The focused laser electric field

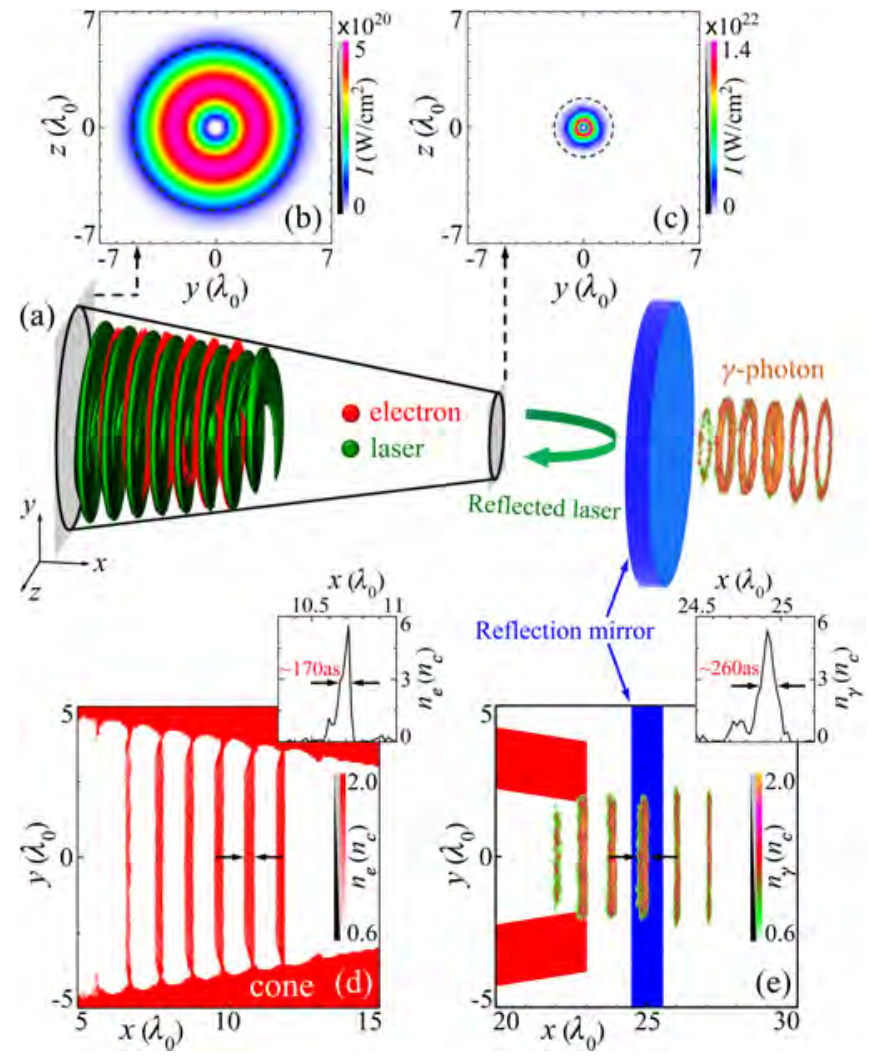

FIG. 1. (a) Schematic of attosecond $\gamma$-photon pulse train$\mathrm{s}$ generation from the laser-driven cone-foil compound target. A CP LG laser pulse (green isosurface) incident from the left side of the simulation box irradiates a cone target (black frame), which further focuses the laser pulse. Electrons (red rings) are extracted from the cone walls and accelerated by the laser fields, travelling along the $x$ direction. Later, the focusing laser pulse is reflected by a plasma mirror pulsed on the surface of a dense foil (blue plate) adjacent to the cone and collides head-on with the dense energetic attosecond electron bunches, resulting in the generation of ultra-bright multi$\mathrm{MeV}$ attosecond $(\lesssim 260$ as $) \gamma$-photon pulse trains. The spatial distributions of the laser intensity in the $y-z$ plane for the incident pulse (b) and in-cone pulse (c) are shown, where the black-dashed circles indicate the left and right (apex) aperture profiles of the cone, respectively. Density distributions of electrons (d) at $t=14 T_{0}$ and $\gamma$-photons (e) at $t=30 T_{0}$.

shows spatially periodic, alternating positive and negative structures, which is shown in supplementary material. This plays a key role in the formation of isolated electron atto-bunches (See details in supplementary material). The maximum energy gained by the electrons can be calculated as

$$
\varepsilon_{m} \sim e \bar{E}_{m} l_{d}
$$

where $\bar{E}_{m} \sim \bar{E}_{x}$ is the average accelerating gradien$\mathrm{t}$ and $l_{d}$ is the accelerating distance. One can derive the longitudinal electric field of $E_{x}=2 \sqrt{2} E_{0} \exp (1 / 2-$ $\left.r^{2} / \sigma_{0}^{2}\right)\left(1-r^{2} / \sigma_{0}^{2}\right) / k \sigma_{0}$ from the Possion equation $(\nabla$. $\mathbf{E}=0$ ), and obtain its average amplitude of $\bar{E}_{x} \sim$ 

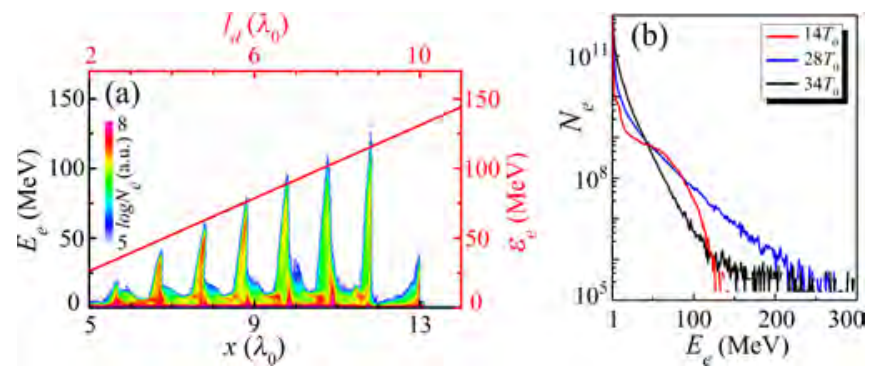

FIG. 2. (a) The spatial distribution of the electron energy along the $x$-direction at $t=14 T_{0}$, where the red line shows the energy gain $\left(\varepsilon_{e}\right)$ of electrons predicted using Eq. (1). (b) Temporal evolution of the electron energy spectrum.

$0.4\left|E_{x}\right|_{m} \approx 0.8 \sqrt{2} \exp (1 / 2) E_{0} / k \sigma_{0}$. In our configuration, $E_{x}$ is greatly increased due to the strong focusing of the laser pulse in plasmas, so that the peak energy of accelerated electrons can be rewritten as $\varepsilon_{m} \sim$ $0.8 \sqrt{2} \exp (1 / 2) \bar{a}_{f o c} m_{e} c^{2} l_{d} / \sigma_{0}$, where $\bar{a}_{f o c}$ is the average normalized amplitude of the focused laser. This indicates that the electron energy gain increases with $l_{d}$, which is validated by our PIC simulations as shown in Fig. 2(a). By taking $\bar{a}_{f o c} \sim\left(a_{m}+a_{0}\right) / 2 \approx 48$ and $l_{d} \approx 20 \lambda_{0}$ for example, the maximum electron energy is about $260 \mathrm{MeV}$, which agrees well with our simulation results, as shown in Fig. 2(b). Finally, dense isolated electron atto-bunches with energy of hundreds of $\mathrm{MeV}$ are generated with a spacing of $\sim 1 \lambda_{0}$ between the bunch trains. This effective energetic electron atto-bunch source is very beneficial for ultrashort $\gamma$-ray pulse generation.

In the stage of laser-driven electron acceleration, although the electrons can be accelerated to several hundreds of $\mathrm{MeV}$ and the LG laser intensity can be enhanced to $10^{22} \mathrm{~W} / \mathrm{cm}^{2}$, high-energy photon emission is still very limited in this process. This is because the contribution from the transverse electric field $\mathbf{E}_{\perp}$ to the quantum radiation-dominated parameter $\eta$ can be almost fully canceled out by the $\mathbf{v} \times \mathbf{B}$ term in the co-propagating stage, resulting in $\eta \rightarrow 0$, where ${ }^{41,42}$

$$
\eta=\left(\gamma_{e} / E_{s}\right)\left|\mathbf{E}_{\perp}+\mathbf{v} \times \mathbf{B}\right| .
$$

Here, $\gamma_{e}$ is the Lorentz factor of the electrons, $\mathbf{E}_{\perp}$ is the electric field perpendicular to the electron velocity $\mathbf{v}, E_{s}=m_{e}^{2} c^{3} / e \hbar$ is the critical field of $\mathrm{QED}^{43}$ and $\hbar$ is the reduced Planck constant. For efficient high-energy radiation emission, $\eta \gtrsim 0.1$ is essential ${ }^{27,44,45}$.

When the tightly-focused laser pulse reflects from the dense foil, it subsequently collides with the highenergy electrons to excite the NCS process. At this head-on collision stage, the quantum parameter is $\eta \approx$ $2 \gamma_{e}\left|\mathbf{E}_{\perp}\right| / E_{s}$, which becomes much larger than 0.1 in our configuration, so that high-energy $\gamma$-photons emission is significantly enhanced. The power of the radiation emitted by the electron can be written as $P_{r a d} \approx$ $2 e E_{s} c \alpha_{f} \eta^{2} g(\eta) / 3$, where $\alpha_{f}$ is the fine-structure constan$\mathrm{t}, g(\eta)=\left(3 \sqrt{3} / 2 \pi \eta^{2}\right) \int_{0}^{\infty} F(\eta, \chi) d \chi$ is considered as the
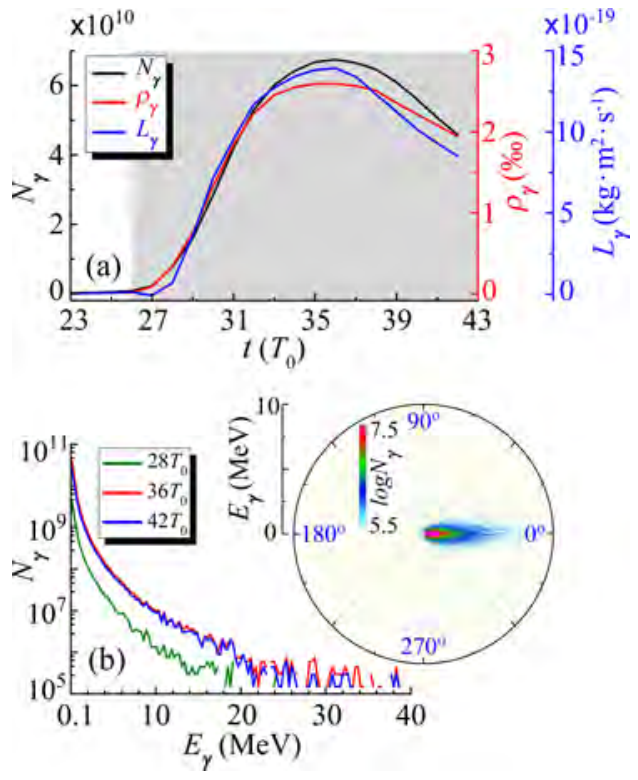

FIG. 3. (a) Temporal evolution of the photon yield $\left(N_{\gamma}\right)$, energy conversion efficiency $\left(\rho_{\gamma}\right)$, and angular momentum $\left(L_{\gamma}\right)$ of $\gamma$-photon beams, where the gray area shows the photon emission in the collision stage. (b) The angular-energy distribution of $\gamma$-photons and the $\gamma$-photon energy spectra at different times. Note that here we only consider the photons with energy $>0.1 \mathrm{MeV}$.

radiation correction induced by QED effects ${ }^{41,42}$. The radiation power estimated is $\sim 2 \mathrm{TW}$, which agrees reasonably with our PIC simulation result of 3TW. Figure 3(a) presents the laser energy conversion. The energy conversion efficiency from the laser to $\gamma$-photons is as high as $2.6 \%$, which is tens-fold higher than that obtained by other plasma-based mechanisms ${ }^{11,12,46}$. This suggest$\mathrm{s}$ that our scheme offers an efficient approach to obtain attosecond $\gamma$-photon pulses with intensity of $10^{20} \mathrm{~W} / \mathrm{cm}^{2}$ in the multi-TW level. This in turn may enable promising avenues for studying attosecond-pump/probe experiments in a high intensity and multi-MeV range.

The definition of the beam angular momentum with respect to the $x$-axis can be expressed as

$$
\left.L\right|_{x}=\sum_{i}\left|\mathbf{r}_{i} \times \mathbf{p}_{i}\right|_{x} \propto N \bar{r} \bar{p}
$$

where the subscript $i$ denotes serial number of individual particles, and $N, \bar{r}, \bar{p}$ are the total number, average offaxis radius, and average transverse momentum, respectively. The $\gamma$-photon emission from the NCS of energetic electrons, where they are emitted in the direction of electron motion, whose momentum originates from the parent electrons and can be scaled as $p_{\gamma} \propto \eta^{2} g(\eta)$. One can thus estimate the beam angular momentum of $\gamma$-photons as $L_{\gamma}=\sum_{i}\left|\mathbf{r}_{\gamma i} \times \mathbf{p}_{\gamma i}\right|_{x} \propto N_{\gamma} \bar{r}_{\gamma} \eta^{2} g(\eta)$, where $N_{\gamma}$ and $\bar{r}_{\gamma}$ are the yield and average off-axis radius of $\gamma$-photons. As expected, the $\gamma$-photon emission can be ignored with $\eta \rightarrow 0$ at the first stage. However, the efficiency of $\gamma$ photons emission becomes very high with the significant 
increase of $\eta$. Both the angular momentum and energy conversion efficiency from the laser to $\gamma$-photons increase accordingly, as presented in Fig. 3(a).

In our configuration, the quantum parameter $\eta$ is as high as 0.3 and the energy of the generated photons ${ }^{47}$ can be evaluated to be up to $\hbar \omega \approx 0.44 \eta \gamma_{e} m_{e} c^{2} \sim$ $34 \mathrm{MeV}$, which agrees well with the energy spectra in Fig. 3(b). Considering a single pulse from the train, gives a photon yield of $10^{9}$ at $1 \mathrm{MeV}$, a full width at half maximum (FWHM) duration of $\sim 260 \mathrm{as}$, a source cross-section equal to $\sim 1 \mu \mathrm{m}^{2}$, and a FWH$\mathrm{M}$ divergence of $0.2 \times 0.2 \mathrm{rad}^{2}$. This results in an $\gamma$ photon source with an ultrahigh peak brilliance of $\sim$ $10^{23}$ photons $/ \mathrm{s} / \mathrm{mm}^{2} / \mathrm{mrad}^{2} / 0.1 \% \mathrm{BW}$ and an ultrashort duration of $<550$ as per pulse at $\mathrm{MeV}$ energies. Here the brilliance of the $\gamma$-ray is much higher than that obtained in current experiments ${ }^{11,12}$. Such ultra-brilliant $\mathrm{MeV}$ attosecond $\gamma$-photon beams with desirable angular momentum would offer exciting new capabilities and opportunities for diverse studies ${ }^{1-4}$, such as studying nuclear phenomena and structure, discovering new particles and examining their underlying properties.

In our proposed scheme, one can manipulate the emission of $\gamma$-photons and their angular momentum by changing the laser and target parameters. We first discuss the effect of the laser intensity, where we keep all of other parameters unchanged but vary the normalized laser amplitude from $a_{0}=10$ to 40 . It is shown that $\gamma$-photon emission from energetic electrons via the NCS process increases significantly with the laser intensity due to $\eta \propto \gamma_{e}\left|\mathbf{E}_{\perp}\right|$, which is verified by the PIC simulation results in Fig. 4(a). By using a laser with hundreds of TW power, with $a_{0}=40$ for example, ultra-brilliant high angular momentum $\gamma$-photon beams can be generated efficiently with a total yield of $2.3 \times 10^{12}$ and a high energy conversion efficiency of $5.8 \%$. In addition, we find that the yield of $\gamma$-photons scales as $N_{\gamma} \propto a_{0}^{3}$ from numerical simulations. Thus we can obtain the angular momentum scaling of photon beams $L_{\gamma} \propto N_{\gamma} \eta^{2} g(\eta) \propto a_{0}^{\sim 13 / 3}$ in this case, which is in good agreement with our simulation results presented in Fig. 4(a).

We also investigate the effect of the aperture size of the cone apex on the photon emission. Figure 4(b) presents simulation results for which $r_{0}$ is varied in the range from $1 \lambda_{0}$ to $3.5 \lambda_{0}$ while all other parameters are unchanged. The results show that a smaller apex aperture is beneficial for $\gamma$-photon emission. For example, by using a cone target of $r_{0}=1 \lambda_{0}$, high-yield $\left(1.35 \times 10^{11}\right) \gamma$-photons with high angular momentum $\left(2.4 \times 10^{-18} \mathrm{~kg} \cdot \mathrm{m}^{2} \cdot \mathrm{s}^{-1}\right)$ and energy conversion efficiency $(5.1 \% 0)$ are emitted. Conversely, when increasing the apex radius to $r_{0}=3 \lambda_{0}$, both yield and laser energy conversion efficiency decrease significantly, and a smaller degree of total photon angular momentum transfer occurs. This is attributed to the focusing effect of the laser pulse in the conical plasma, where the laser intensity can be approximately scaled as $I \propto \sim 1 / r_{0}^{2}$. However, when further increasing the radius to, e.g. $r_{0}=3.5 \lambda_{0}$, the photon emission will change little
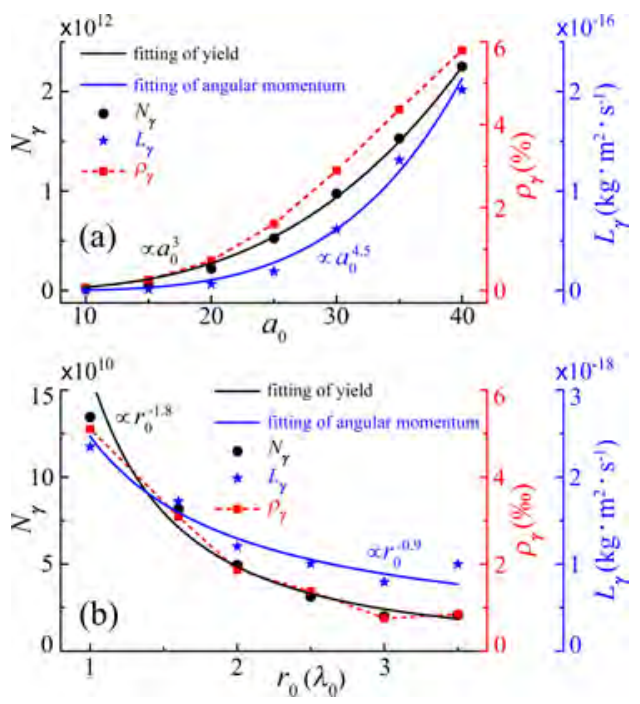

FIG. 4. Scaling of the photon yield ( $N_{\gamma}$, black circles), the laser energy conversion efficiency ( $\rho_{\gamma}$, red squares), and the total photon angular momentum $\left(L_{\gamma}\right.$, blue stars) with (a) the laser electric field amplitude $a_{0}$ and (b) the apex aperture radius $r_{0}$ of the cone. Here, the black curves indicate the fitting of the photon yield and the blue curves present the fitting results of angular momentum.

because the influence of cone structure becomes limited and similar to a channel for $r_{0} \gtrsim \sigma_{0}$. The corresponding angular momentum of the photon beam is comparable to that in the case of $r_{0}=2.5 \lambda_{0}$. This is attributed to the relation $L_{\gamma} \propto N_{\gamma} \bar{r}_{\gamma}$, which depends upon not only the photon yield but also the off-axis radius. The angular momentum of photon beams can therefore be approximated as $L_{\gamma} \propto r_{0}^{-0.8}$ with $N_{\gamma} \propto r_{0}^{-1.8}$ based upon the PIC simulations for $r_{0}<\sigma_{0}$. This is reasonably close to the simulation results in Fig. 4(b). Overall, our scheme provides a practical and efficient way to optically control ultra-bright attosecond $\gamma$-rays emission by changing the laser intensity and target structure. This approach could be used to control angular momentum of ultrashort $\gamma$-ray radiation pulses in future experiments.

In conclusion, we have shown that a train of ultrabright, attosecond, energetic $\gamma$-ray pulses can be efficiently generated by NCS via the intensity enhanced laser pulse colliding with energetic electron atto-bunches. Moreover, one can manipulate the yield, energy conversion efficiency and angular momentum of $\gamma$-photon beam$\mathrm{s}$ by tuning the parameters of laser intensity and cone structure. Such unique $\mathrm{MeV}$ attosecond $\gamma$-ray sources have many advantages over existing sources, which may open up new research avenues in ultrafast science and potentially also be applied in industry and medicine.

See supplementary material for more details on the spatial distribution of the focused circularly-polarized Laguerre-Gaussian laser pulse and its role in the formation of attosecond electron bunches.

The authors wish to acknowledge supports from the National Science Foundation of China (Grant Nos. 
11721091, 11655002, 11774227, and 11622547), the National Basic Research Program of China (Grant No. 2013CBA01504), the Ministry of Science and Technology of China for an International Collaboration Project (Grant No. 2014DFG02330), the Science and Technology Commission of Shanghai Municipality (Grant No. 16DZ2260200), Hunan Provincial Natural Science Foundation of China (Grant No. 2017JJ1003), and a Leverhulme Trust Grant at the University of Strathclyde. The EPOCH code was in part developed by the UK EPSRC grants EP/G056803/1. All simulations were performed on the $\Pi$ supercomputer at Shanghai Jiao Tong University.

${ }^{1}$ T. Brabec and F. Krausz, Rev. Mod. Phys. 72, 545 (2000).

${ }^{2}$ F. Krausz and M. Ivanov, Rev. Mod. Phys. 81, 163 (2009).

${ }^{3}$ A. Baltuška, T. Udem, M. Uiberacker, M. Hentschel, E. Goulielmakis, C. Gohle, R. Holzwarth, V. Yakovlev, A. Scrinzi, and T. W. Hänsch, Nature 421, 611 (2003).

${ }^{4}$ P. á. Corkum and F. Krausz, Nat. Phys. 3, 381 (2007).

${ }^{5}$ U. Teubner and P. Gibbon, Rev. Mod. Phys. 81, 445 (2009).

${ }^{6}$ G. Sansone, L. Poletto, and M. Nisoli, Nat. Photon. 5, 655 (2011).

${ }^{7}$ M. Yeung, S. Rykovanov, J. Bierbach, L. Li, E. Eckner, S. Kuschel, A. Woldegeorgis, C. Rödel, A. Sävert, and G. Paulus, Nat. Photon. 11, 32 (2017).

${ }^{8}$ M. Drescher, M. Hentschel, R. Klenberger, and M. Ulberacker, Nature 419, 802 (2002).

${ }^{9}$ K. W. D. Ledingham, P. McKenna, and R. P. Singhal, Science 300, 1107 (2003).

${ }^{10}$ S. Cipiccia, M. R. Islam, B. Ersfeld, R. P. Shanks, E. Brunetti, G. Vieux, X. Yang, R. C. Issac, S. M. Wiggins, G. H. Welsh, M.-P. Anania, D. Maneuski, R. Montgomery, G. Smith, M. Hoek, D. J. Hamilton, N. R. C. Lemos, D. Symes, P. P. Rajeev, V. O. Shea, J. M. Dias, and D. A. Jaroszynski, Nat. Phys. 7, 867 (2011).

${ }^{11}$ K. T. Phuoc, S. Corde, C. Thaury, V. Malka, A. Tafzi, J. P. Goddet, R. C. Shah, S. Sebban, and A. Rousse, Nat. Photon. 6, 308 (2012).

${ }^{12}$ G. Sarri, D. J. Corvan, W. Schumaker, J. M. Cole, A. Di Piazza, H. Ahmed, C. Harvey, C. H. Keitel, K. Krushelnick, S. P. D. Mangles, Z. Najmudin, D. Symes, A. G. R. Thomas, M. Yeung, Z. Zhao, and M. Zepf, Phys. Rev. Lett. 113, 224801 (2014).

${ }^{13}$ W. Yan, C. Fruhling, G. Golovin, D. Haden, J. Luo, P. Zhang, B. Zhao, J. Zhang, C. Liu, M. Chen, S. Chen, S. Banerjee, and D. Umstadter, Nat. Photon. 11, 514 (2017).

${ }^{14}$ H. He, M. E. J. Friese, N. R. Heckenberg, and H. RubinszteinDunlop, Phys. Rev. Lett. 75, 826 (1995).

${ }^{15}$ C. Thaury, E. Guillaume, S. Corde, R. Lehe, M. Le Bouteiller, K. Ta Phuoc, X. Davoine, J. M. Rax, A. Rousse, and V. Malka, Phys. Rev. Lett. 111, 135002 (2013).

${ }^{16}$ Y. Taira, T. Hayakawa, and M. Katoh, Sci. Rep. 7, 5018 (2017).

${ }^{17}$ R. Ruffini, G. Vereshchagin, and S.-S. Xue, Phys. Rep. 487, 1 (2010).

${ }^{18}$ M. Katoh, M. Fujimoto, H. Kawaguchi, K. Tsuchiya, K. Ohmi, T. Kaneyasu, Y. Taira, M. Hosaka, A. Mochihashi, and Y. Takashima, Phys. Rev. Lett. 118, 094801 (2017).

${ }^{19}$ V. Petrillo, G. Dattoli, I. Drebot, and F. Nguyen, Phys. Rev. Lett. 117, 123903 (2016).

${ }^{20}$ C. Liu, B. Shen, X. Zhang, Y. Shi, L. Ji, W. Wang, L. Yi, L. Zhang, T. Xu, Z. Pei, and Z. Xu, Phys. Plasmas 23, 093120 (2016).

${ }^{21}$ Y.-Y. Chen, J.-X. Li, K. Z. Hatsagortsyan, and C. H. Keitel, arXiv:1802.04748.
${ }^{22}$ J.-X. Li, K. Z. Hatsagortsyan, B. J. Galow, and C. H. Keitel, Phys. Rev. Lett. 115, 204801 (2015).

${ }^{23}$ J. Yu, Z. Najmudin, R. Hu, T. Tajima, H. Lu, and X. Yan, arXiv:1705.07075.

${ }^{24}$ T. Nakamura, J. K. Koga, T. Z. Esirkepov, M. Kando, G. Korn, and S. V. Bulanov, Phys. Rev. Lett. 108, 195001 (2012).

${ }^{25}$ T. P. Yu, A. Pukhov, Z. M. Sheng, F. Liu, and G. Shvets, Phys. Rev. Lett. 110, 045001 (2013).

${ }^{26}$ M. Vranic, J. L. Martins, J. Vieira, R. A. Fonseca, and L. O. Silva, Phys. Rev. Lett. 113, 134801 (2014).

${ }^{27}$ X.-L. Zhu, T.-P. Yu, Z.-M. Sheng, Y. Yin, I. C. E. Turcu, and A. Pukhov, Nat. Commun. 7, 13686 (2016).

${ }^{28}$ M. Chen, J. Luo, F.-Y. Li, F. Liu, Z.-M. Sheng, and J. Zhang, Light Sci. Appl. 5, e16015 (2016).

${ }^{29}$ A. Gonoskov, A. Bashinov, S. Bastrakov, E. Efimenko, A. Ilderton, A. Kim, M. Marklund, I. Meyerov, A. Muraviev, and A. Sergeev, Phys. Rev. X 7, 041003 (2017).

${ }^{30}$ C. Bula, K. T. McDonald, E. J. Prebys, C. Bamber, S. Boege, T. Kotseroglou, A. C. Melissinos, D. D. Meyerhofer, W. Ragg, D. L. Burke, R. C. Field, G. Horton-Smith, A. C. Odian, J. E. Spencer, D. Walz, S. C. Berridge, W. M. Bugg, K. Shmakov, and A. W. Weidemann, Phys. Rev. Lett. 76, 3116 (1996).

${ }^{31}$ F. Mackenroth and A. Di Piazza, Phys. Rev. A 83, 032106 (2011).

${ }^{32}$ T. D. Arber, K. Bennett, C. S. Brady, A. Lawrence-Douglas, M. G. Ramsay, N. J. Sircombe, P. Gillies, R. G. Evans, H. Schmitz, and A. R. Bell, Plasma Phys. Controlled Fusion 57, 113001 (2015).

${ }^{33}$ C. P. Ridgers, J. G. Kirk, R. Duclous, T. G. Blackburn, C. S. Brady, K. Bennett, T. D. Arber, and A. R. Bell, J. Comput. Phys. 260, 273 (2014).

${ }^{34}$ L. Allen, M. W. Beijersbergen, R. Spreeuw, and J. Woerdman, Phys. Rev. A 45, 8185 (1992).

${ }^{35} \mathrm{M}$. W. Beijersbergen, L. Allen, H. Van der Veen, and J. Woerdman, Opt. Commun. 96, 123 (1993).

${ }^{36}$ Y. Shi, B. Shen, L. Zhang, X. Zhang, W. Wang, and Z. Xu, Phys. Rev. Lett. 112, 235001 (2014).

${ }^{37}$ J. Vieira, R. M. G. M. Trines, E. P. Alves, R. A. Fonseca, J. T. Mendona, R. Bingham, P. Norreys, and L. O. Silva, Nat. Commun. 7, 10371 (2016).

${ }^{38}$ A. G. MacPhee, L. Divol, A. J. Kemp, K. U. Akli, F. N. Beg, C. D. Chen, H. Chen, D. S. Hey, R. J. Fedosejevs, R. R. Freeman, M. Henesian, M. H. Key, S. Le Pape, A. Link, T. Ma, A. J. Mackinnon, V. M. Ovchinnikov, P. K. Patel, T. W. Phillips, R. B. Stephens, M. Tabak, R. Town, Y. Y. Tsui, L. D. Van Woerkom, M. S. Wei, and S. C. Wilks, Phys. Rev. Lett. 104, 055002 (2010).

${ }^{39}$ X. L. Zhu, Y. Yin, T. P. Yu, F. Q. Shao, Z. Y. Ge, W. Q. Wang, and J. J. Liu, New J. Phys. 17, 053039 (2015).

${ }^{40}$ L. L. Ji, J. Snyder, A. Pukhov, R. R. Freeman, and K. U. Akli, Sci. Rep. 6, 23256 (2016).

${ }^{41}$ V. I. Ritus, J. Russ. Laser Res. 6, 497 (1985).

42 J. G. Kirk, A. R. Bell, and I. Arka, Plasma Phys. Controlled Fusion 51, 085008 (2009).

${ }^{43}$ J. Schwinger, Phys. Rev. 82, 664 (1951).

${ }^{44}$ T. G. Blackburn, C. P. Ridgers, J. G. Kirk, and A. R. Bell, Phys. Rev. Lett. 112, 015001 (2014).

${ }^{45}$ D. J. Stark, T. Toncian, and A. V. Arefiev, Phys. Rev. Lett. 116, 185003 (2016).

${ }^{46}$ L. Yi, A. Pukhov, P. Luu-Thanh, and B. Shen, Phys. Rev. Lett. 116, 115001 (2016).

${ }^{47}$ A. R. Bell and J. G. Kirk, Phys. Rev. Lett. 101, 200403 (2008). 